\title{
High spatial resolution identification of hematoma in inhomogeneous head phantom using broadband fNIR system
}

\author{
E. Sultan ${ }^{1^{*}}$ (D, A. H. Gandjbakhche ${ }^{3}$, K. Pourrezaei ${ }^{4}$ and A. S. Daryoush ${ }^{2}$
}

\author{
*Correspondence: \\ ek.sultan@paaet.edu.kw \\ ${ }^{1}$ Dept. of Electronics \\ Engineering, College \\ of Technological Studies, \\ PAAET, Kuwait, Kuwait \\ Full list of author information \\ is available at the end of the \\ article
}

\begin{abstract}
This paper presents a novel method for early detection of hematomas using highly sensitive optical fNIR imaging methods based on broadband photon migration. The NIR experimental measurements of inhomogeneous multi-layer phantoms representing human head are compared to 3D numerical modeling over broadband frequencies of 30-1000 MHz. A finite element method (FEM) simulation of the head phantom are compared to measurements of insertion loss and phase using custom-designed broadband free space optical transmitter (Tx) and receiver (Rx) modules that are developed for photon migration at wavelengths of $670 \mathrm{~nm}, 795 \mathrm{~nm}, 850 \mathrm{~nm}$, though results of $670 \mathrm{~nm}$ are discussed here. Standard error is used to compute error between 3D FEM modeling and experimental measurements by fitting experimental data to the $a \sqrt{\text { frequency }}+b$. Error results are shown at narrowband and broadband frequency modulation in order to have confidence in 3D numerical modeling. A novel method is established here to identify presence of hematoma based on first and second derivatives of changes in insertion loss and phase $(\Delta \mathrm{L}$ and $\Delta I P)$, where frequency modulated photons sensitive to different sizes of hematoma is identified for wavelength of $670 \mathrm{~nm}$. The high accuracy of this comparison provides confidence in optical bio-imaging and its eventual application to TBI detection.
\end{abstract}

Keywords: $\mathrm{fNIR}, \mathrm{TBI}, \mathrm{FEM}, \mathrm{DE}, \mathrm{COMSOL}$, Optical transmitter Tx, Optical receiver Rx, Triwavelength VCSEL, PDW, IL, IP

\section{Introduction}

Bio-imaging techniques have shown a great deal of confidence in improving the diagnosis and clinical care for civilians and military personnel, who experience a traumatic brain injury (TBI) due to sports, auto accidents, or explosions from improvised explosive device (IED) [1]. Injuries of this caliber can lead to temporary or permanent impaired brain functionality. Commonly categorized as either an open or closed injuries; scalp and skull penetration due to a sharp object/bullet are seen in open, while closed injuries occur when the head is shaken violently due to acoustic boom. Both can lead to mild to severe TBI, causing sheared or torn brain axons, blood vessels, or both [2]. Most undiagnosed TBI injuries are closed head injures and without fractures. Another type of injury to the brain is the development of a hematoma. Disrupted blood vessels that leak into the layers of dura mater or between the skull and dura mater, might lead to epidural

0 The Author(s) 2018. This article is distributed under the terms of the Creative Commons Attribution 40 International License (http://creativecommons.org/licenses/by/4.0/), which permits unrestricted use, distribution, and reproduction in any medium, provided you give appropriate credit to the original author(s) and the source, provide a link to the Creative Commons license, and indicate if changes were made. The Creative Commons Public Domain Dedication waiver (http://creativecommons.org/publicdomain/zero/1.0/) applies to the data made available in this article, unless otherwise stated. 
or subdural hematomas [3]. It can be medically confirmed by CT scan or MRI imaging techniques [4]. Due to lack of mobility and high cost of these imaging modalities, optical imaging using functional near infrared (fNIR) is being developed by understanding the relation between migrated photons and biological media.

Photons in the near-IR (NIR) region are particles that have both absorption and scattering properties when interacted with biological media such as tissue, cells, or organs [5]. Biological media have optical properties that include both reflection and absorption characteristics [5]. Photons suffer broadening and eventual decay as it travels because of multiple scattering and absorption when penetrated and traveled through bio-media. Propagation of these photons within bio-media depends on the media optical properties of absorption and scattering which is defined by particles within cells, cell organelles, and fiber structure [6]. Optical parameters of the tissue depend on both absorption and scattering of photons which reveals information related to hemoglobin and water concentration [7]. This information is used to determine an important aspect of biological functionality that is related to concentration of oxygen/de-oxygenated level in hemoglobin and the amount of water when photons have penetrated and traveled in biomedia and this method of imaging is called functional near infrared (fNIR) imaging.

The fNIR based spectroscopic studies of biological media have shown the capability of measuring the absorption and scattering sensitivity of water, oxygenated, and de-oxygenated hemoglobin at different wavelengths. In that regard any impact that will lead to disorder the normal functionally of the neurons of brain could be then registered by disorder in the absorption of oxygenated or de-oxygenated hemoglobin using diffused photon NIR (DPNIR) [8]. The location and percentage of oxygen absorbed in the brain can be related to different physiological activities through fNIR system. Measuring the reduction in de-oxygenated hemoglobin or increase in oxygenated hemoglobin leads to an increase in oxygenated blood volume and having this localized information related to hematoma detection [9], would aid in earlier diagnosis. Resulting is faster, more efficient and cost-effective treatment. Moreover, early detection and efficacy of this proposed treatment could be compared using fNIR imaging, by monitoring the performance of human brain regions over time.

This paper will examine for the first time the ability of a broadband free-space fNIR in detecting high optical absorbing media that resembles a human head with an embedded hematoma and studies the spatial resolution detection of a hematoma. The concept followed in this paper is based on the assumption that a hematoma is generated after a blast or accident, and that broadband fNIR system is advantageous in detecting smaller hematomas. Focusing mainly on hematomas, the method of detection consists of both numerical modeling and an experimental measurement that follows the frequency modulated photon concept traveling in multi-layer inhomogeneous human brain phantoms. It should be noted that these phantoms resembles a human head, which is embedded with a hematoma at an outer layer of the cortex. A conceptual representation of human head phantom and optical system setup is shown in Fig. 1. Optical transmitter (Tx) and receivers $(\mathrm{Rx})$ are strategically located on head phantom across the hematoma. This study is part of early detection of traumatic brain injury (TBI) using a helmet mounted functional near infrared (fNIR) device [10, 11]. A 3D FEM modeling of diffusion equation (DE) for inhomogeneous head phantoms is employed to quantify hematoma sizes 


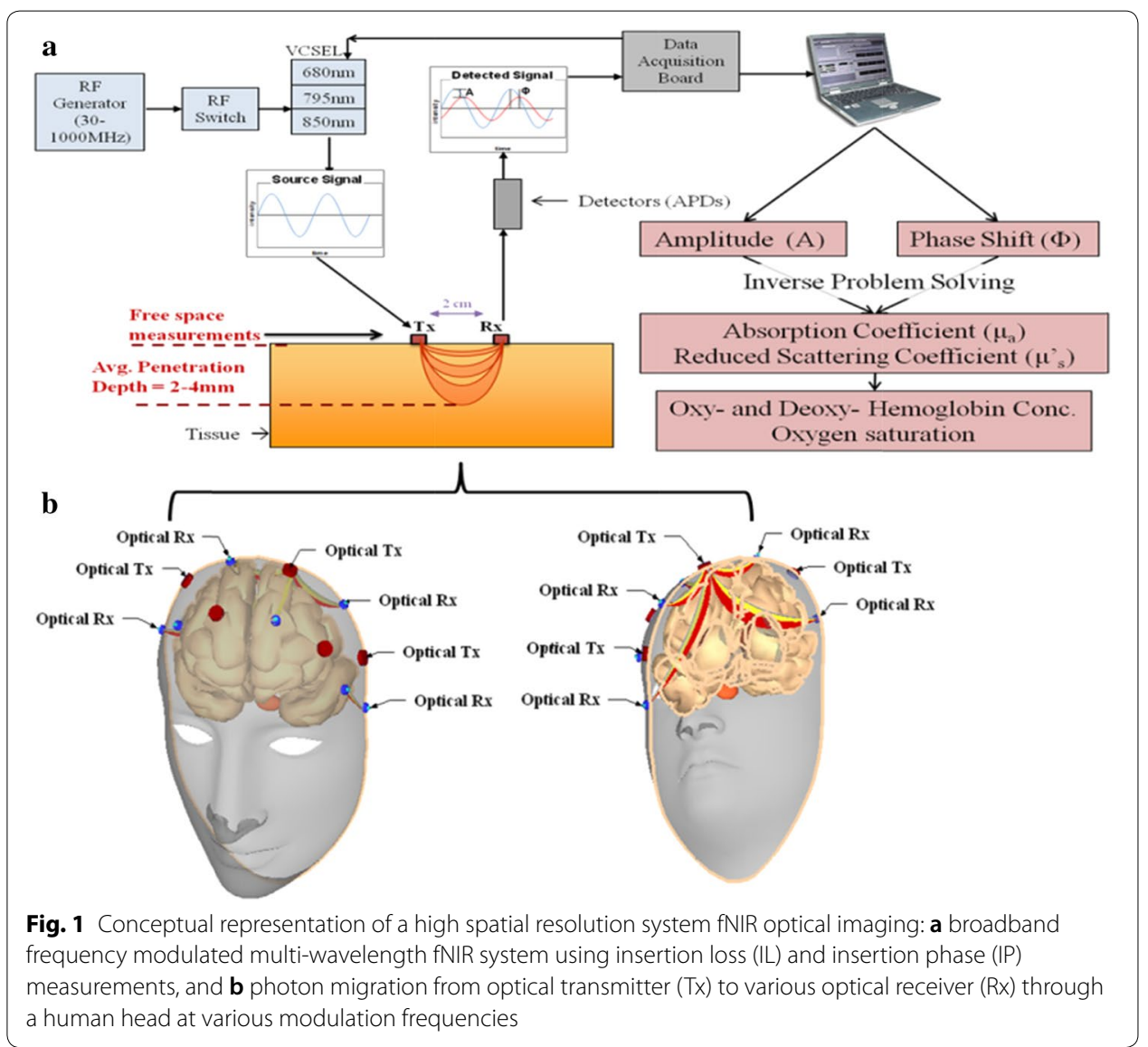

approaching sub-centimeter spatial resolutions. First, this paper presents comparison between 3D FEM and experimental result at wavelength of $670 \mathrm{~nm}$ to quantify accuracy of model fitting by comparing insertion loss and phase from 3D modeling and measurements results that are curve fitted over various modulation frequency bandwidths. Second, a signal processing method is proposed by taking advantage of first and second derivative of the differential measurements of IL between cortex with and without occlusion for detection hematoma as photons interact with different sizes of occlusion representing hematoma.

\section{Numerical modeling and experimental methods}

Broadband frequency modulation method has proven high efficiency when applied to functional optical imaging systems on homogenous and inhomogeneous biological media, and proven to present with high optical parameter extraction accuracy as shown in previous publications $[12,13]$. The method is to be extended here to monitor the early formalization of hematoma as a sign of TBI. An optical system using broadband modulation is used along with three layers of phantom, embedded with different sizes of occlusion. After an injury, a hematoma can develop slowly, in relation to time. Therefore an occlusion of different sizes in diameter is used for this study to measure the sensitivity of our numerical model and experimental performance in detecting smaller sizes of an occlusion. These occlusions have optical properties similar to the one formed after a 


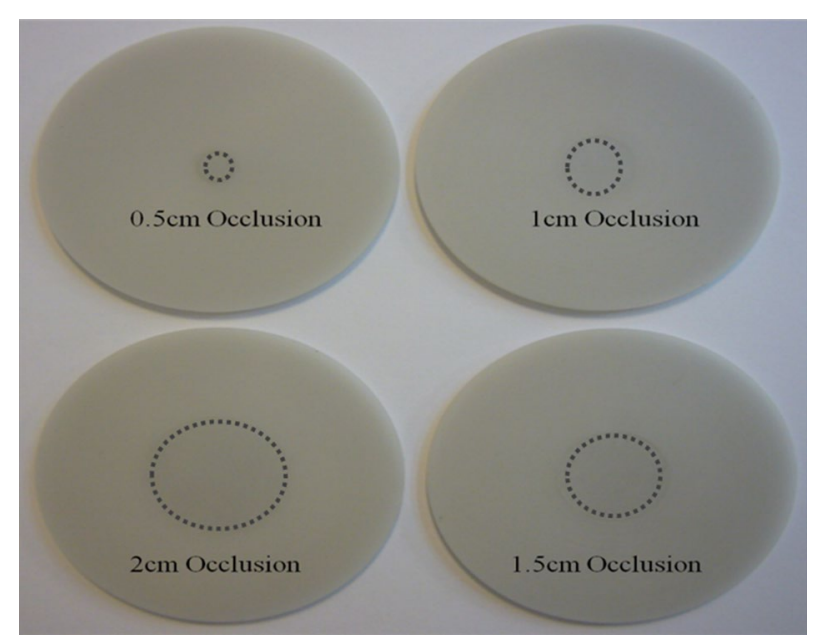

Fig. 2 Images of solid phantoms of different sizes of occlusion embedded in cortex phantom to resemble formation of different sizes of hematoma in cortex. The dashed circles emphasize optical absorption difference between cortex and hematoma

Table 1 Phantom optical parameter properties at wavelength of $670 \mathrm{~nm}$

\begin{tabular}{llllc}
\hline & Diameter $(\mathbf{c m})$ & Thickness $(\mathbf{c m})$ & $\boldsymbol{\mu}_{\boldsymbol{a}}\left(\mathbf{c m}^{-\mathbf{1}}\right)$ & $\boldsymbol{\mu}_{\mathbf{s}}^{\prime}\left(\mathbf{c m}^{-\mathbf{1}}\right)$ \\
\hline Cortex & 5 & 3.5 & 0.35 & 15 \\
Scalp & 5 & 0.3 & 0.15 & 13 \\
Skull & 5 & 0.4 & 0.12 & 8 \\
Occlusion 1 & 0.5 & 0.2 & 0.6 & 18 \\
Occlusion 2 & 1 & 0.2 & 0.6 & 18 \\
Occlusion 3 & 1.5 & 0.2 & 0.6 & 18 \\
Occlusion 4 & 2 & 0.2 & 0.6 & 18 \\
\hline
\end{tabular}

blast or concussion. The phantoms used in the experiments with various occlusions are depicted in Fig. 2, where different sizes of occlusion are designed and embedded in the center of the cortex layer; the optical properties of the occlusions $[14,15]$ are shown in Table 1. Occlusions are designed to start with a diameter of $0.5 \mathrm{~cm}$ and go up to $2 \mathrm{~cm}$, with increments of $0.5 \mathrm{~cm}$, with a height of $0.2 \mathrm{~cm}$. The three layers with the embedded occlusions are then stacked on top of each other and the functional NIR imaging based on broadband frequency modulation is performed at three different wavelengths with a separation of $2 \mathrm{~cm}$, as shown in Fig. 3.

Accurate 3D FEM numerical modeling requires experimental verification of change in amplitude and phase of the incident photon wave that is related to absorption and scattering of the biological media is presented in the frequency domain as insertion loss (IL) and insertion phase (IP). Whereas IL is change in amplitude and IP is change in phase of RF signal modulating NIR photons as they pass through inhomogeneous turbid media. Finite element modeling (FEM) of DE using the commercially available software (multiphysics COMSOL from FEMLab) is preferred over Monte-Carlo simulation of photons because of its good accuracy at a much shorter processing time. The 3D FEM simulation provides graphical or tabular values of DE solution for a given geometry and boundary 


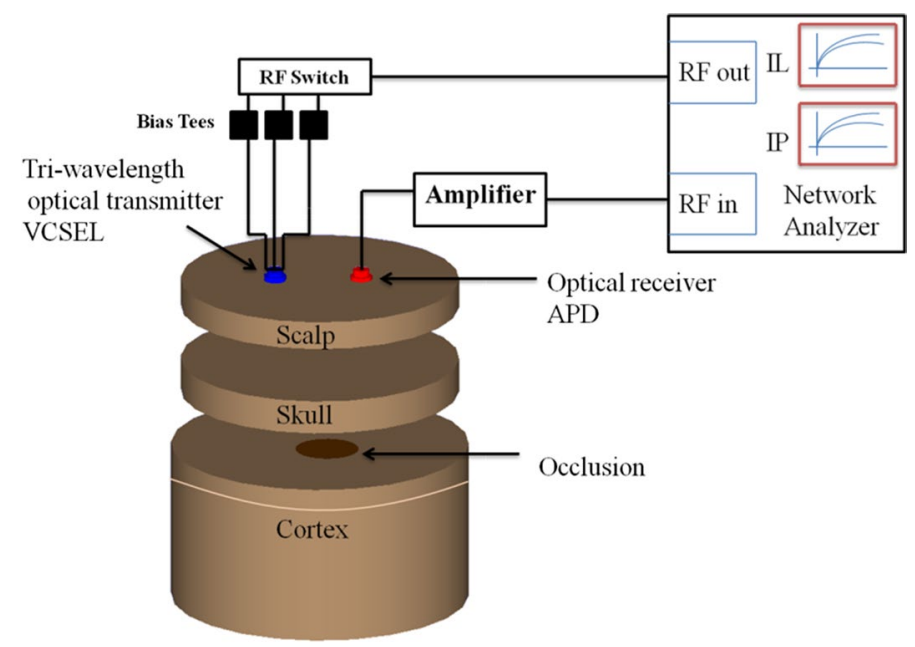

Fig. 3 Optical system and three layer phantoms with embedded occlusion

Table 2 Coefficients and relevant expressions used in Helmholtz sub-domains that govern each layer of human head phantom

\begin{tabular}{lcc}
\hline & Coefficient "c" & Coefficient "a" \\
\hline Layer 1 & $\frac{1}{3\left(\mu_{a 1}+\mu_{s 1}^{\prime}\right)}$ & $\mu_{a 1}$ \\
Layer 2 & $\frac{1}{3\left(\mu_{a 2}+\mu_{s 2}^{\prime}\right)}$ & $\mu_{a 2}$ \\
Layer 3 & $\frac{1}{3\left(\mu_{a 3}+\mu_{s 3}^{\prime}\right)}$ & $\mu_{a 3}$ \\
Occlusion & $\frac{1}{3\left(\mu_{a 4}+\mu_{s 4}^{\prime}\right)}$ & $\mu_{a 4}$
\end{tabular}

conditions [13]. The next section will explain the tools used to model the human head phantom with embedded occlusion that replicates TBI condition.

\section{Finite element method}

The solution of the diffusion equation is modeled in COMSOL through Helmholtz equation for FE numerical analysis [13], where the general form of the DE is:

$$
\nabla(-D \nabla \emptyset(r, t))+\mu_{a} \emptyset(r, t)=S(r, t)
$$

where $\emptyset(r, t)$ is the photon flux, $D=\frac{1}{3\left(\mu_{a}+\mu_{s}^{\prime}\right)}$, and in a frequency modulated NIR system $S(r, t)=S_{O}\left(1+m e^{j \omega t}\right)$ where $m$ is amplitude modulation index at time harmonic modulation frequency of $\omega$. COMSOL representation of the Helmholtz equation is:

$$
-\nabla \cdot(\mathrm{c} \nabla \mathrm{u})+a u=f
$$

Diffusion parameters of inhomogeneous layers are listed in Table 2. It has been in our experience that having mesh structures (cf. Fig. 4) of element sizes of $80 \mathrm{~nm}$ and $1000 \mathrm{~nm}$ in regions around optical $\mathrm{Tx} / \mathrm{Rx}$ and everywhere else respectively would predict an accurate result (e.g., error of less than 5\%) of amplitude and phase change. A 


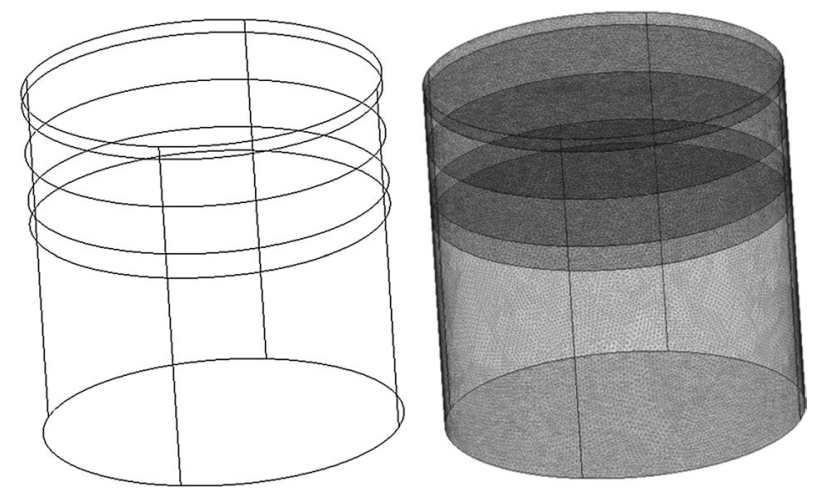

Fig. 4 3D human head layers and the mesh structure applied to simulation

typical computation time of $4 \mathrm{~s}$ for single 2D simulation and $26 \mathrm{~s}$ for single 3D simulation is experienced using an Intel Core $\mathrm{i} 3$ processor PC. These meshing element sizes are also recommended for interfaces (cf. Fig. 4), when multi-layer human head phantoms are modeled [16]. In COMSOL, the boundary condition is set either through Dirichlet or Neumann boundary conditions, where they are set to express the fluence rate, $u$, at desired boundaries. Dirichlet and Neumann boundary conditions are chosen appropriately for air-dielectric (or dielectric-dielectric) and radiation condition in region surrounding phantom. The mathematical representation is expressed [17] as:

$$
\begin{aligned}
& n \cdot\left((c \nabla u)_{1}-\left((c \nabla u)_{2}\right)+q \cdot u=g\right. \\
& h \cdot u=r
\end{aligned}
$$

where $h, r, q$, and $g$ are the boundary coefficients for the phantom modeling. For the diffused photon flux, $\mathrm{h}=1$ and $\mathrm{r}=0$. For the matching boundary of air dielectric and dielectric and dielectric interfaces $\mathrm{q}=0$ and $\mathrm{g}=0$.

\section{Experimental measurement procedures}

The principle of transmitting modulated photons and detecting the traveled photons requires an optical transmitter and optical receiver that operate in a linear region for the desired modulated broadband frequency [12]. The monitoring methods of brain functionality are defined by monitoring different absorption and scattering rates of the oxygenated and de-oxygenated hemoglobin at various wavelengths; therefore, optical transmitters at certain wavelength are desired to quantify insertion loss and phase at various wavelengths to perform inverse problem solution and identify the existence of hematomas in certain regions of the head. The wavelength specifies monitoring the de-oxygenated hemoglobin in the range of 640 to $770 \mathrm{~nm}$ [18] while the oxygenated hemoglobin is in the range of $830-920 \mathrm{~nm}$ [18]. The range of 640 to $770 \mathrm{~nm}$ results in high absorption of photons in the deoxygenated hemoglobin, which means a source of photons in that range will be required to monitor the deoxygenated hemoglobin. While the range of 830 to $920 \mathrm{~nm}$ will result in a high photon absorption in the oxygenated hemoglobin, meaning a source of photons in that range will be required to monitor the oxygenated hemoglobin. 
The overall measurement system consists of Automatic Network Analyzer (Anritsu MS4623B) as an RF source and sensitive RF receiver and broadband optical transmitter various wavelengths and optical receiver modules. The ANA acts as a highly sensitive frequency modulator and demodulator instrument, while the optical transmitter and receiver modules act as broadband modulated photon sources and highly sensitive (i.e., low intensity noise) optical detector. Optical transmitters consisting of tri-wavelengths at $670 \mathrm{~nm}, 850 \mathrm{~nm}$, and $795 \mathrm{~nm}$, are used in this study to monitor peak absorption and cross over absorption of the deoxygenated and oxygenated hemoglobin, respectively. The frequency modulation of the tri-wavelength photon source is controlled by an SP3T RF switch (Hittite HMC245QS16) to drive three high power vertical cavity surface emitting lasers (VCSELs) $(670 \mathrm{~nm}, 795 \mathrm{~nm}, 850 \mathrm{~nm})$. A separate DC biasing control and RF modulating signals is provided for each wavelength through lowpass-highpass Bias-T networks. The VCSEL diode laser (Vixar; Module V3WLM) based optical sources emit high power (output power of about $5 \mathrm{~mW}$ ) Gaussian optical beams with low astigmatism at threshold currents of $8.5 \mathrm{~mA}$ for the $670 \mathrm{~nm}, 9.5 \mathrm{~mA}$ for the $795 \mathrm{~nm}$, and $2.8 \mathrm{~mA}$ for the $850 \mathrm{~nm}$. A printed circuit board has been designed and fabricated using a commercial substrate (FR4), to accommodate all surface mount technology (SMT) components. In absence of PIN Photodiode based commercial high gain optical receivers, APD (Hamamatsu ADP module C5658) have been used along with a built-in trans-impedance amplifier $[12,13]$.

\section{Broadband frequency modulation experimental and simulation results}

Simulated result by COMSOL is done using broadband frequency modulation from 30 to $1000 \mathrm{MHz}$. Figure 4 shows the 3D concept and mesh structure of four layers of phantoms stacked up to form a head phantom with different optical absorption and scattering parameters without any occlusions. The first top two layers are scalp and skull layer, while the last layer is pure cortex layer and these layers have homogenous optical parameters. However, the third layer is a layer of cortex, which could be without any occlusion (i.e., a homogenous optical parameters), as depicted in Fig. 4 or with capability of adding different sized occlusions to resemble a formation of a hematoma in the cortex with inhomogeneous optical parameters (cf. Fig. 6). Identifying the impact of occlusion requires comparison of layers with (occlusion 1-4) against the layer without occlusion (i.e., control). 3D numerical simulation for without occlusion (i.e., control) case is performed at two frequencies of $100 \mathrm{MHz}$ and $1000 \mathrm{MHz}$ and shown in Fig. 5, while experimentally this comparison could be made that as both $\mathrm{Tx}$ and $\mathrm{Rx}$ are pushed away from the occlusion region using translation stages (cf. Figure 2 of reference [13]). These results are later used for identification of high optical activities of occlusions with high absorber.

Different sized occlusions embedded in the third layer of the head phantom are numerically simulated based on mesh structure shown in Fig. 6. Simulations at two frequencies of $100 \mathrm{MHz}$ and $1000 \mathrm{MHz}$ for different sizes of occlusions are shown in Fig. 7, while experimentally broadband measurements for different occlusion sizes and numerical simulation is shown in Fig. 8. Insertion loss and insertion phase measurements at an optical transmitter and receiver separation of $2 \mathrm{~cm}$ around the embedded occlusion, center of the phantoms, is performed along with null location. The null location is considered a control location that is used to compare against change of IL and IP 

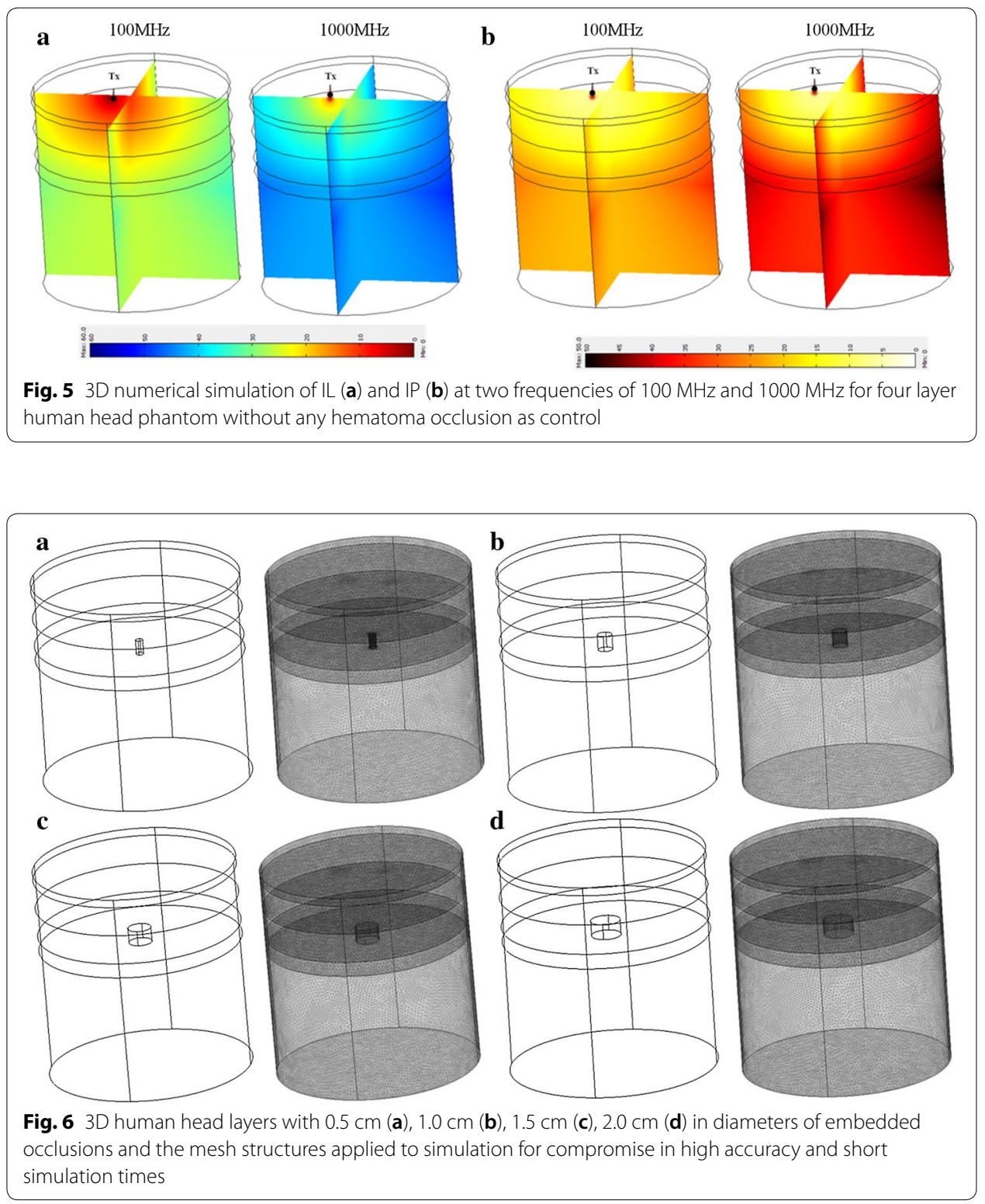

when different sized occlusions are present within the cortex. Experimentally, measured results are done at wavelengths of $670 \mathrm{~nm}$ because of the available information of known phantom optical parameters that was provided from the manufacturer.

At wavelengths of $670 \mathrm{~nm}$, it is observed that the behavior for both IL and IP follows mathematical formulation of $a \sqrt{\text { frequency }}+b$ as expected due to the solution of diffusion equation derived for photons traveling in bio-media [12]. From these results, it is comprehended that the occlusion effect is dominating IL at frequencies greater than $300 \mathrm{MHz}$, while it is not clear at lower frequencies. The location of the occlusion is in the shallow layers of the cortex and because the photon path at high frequency modulation is interacting with the occlusion, the effect of the high absorbing occlusion shows at a frequency-stamp around $300 \mathrm{MHz}$. The important observation is the sensitivity observed by Insertion loss and insertion phase for the smallest 


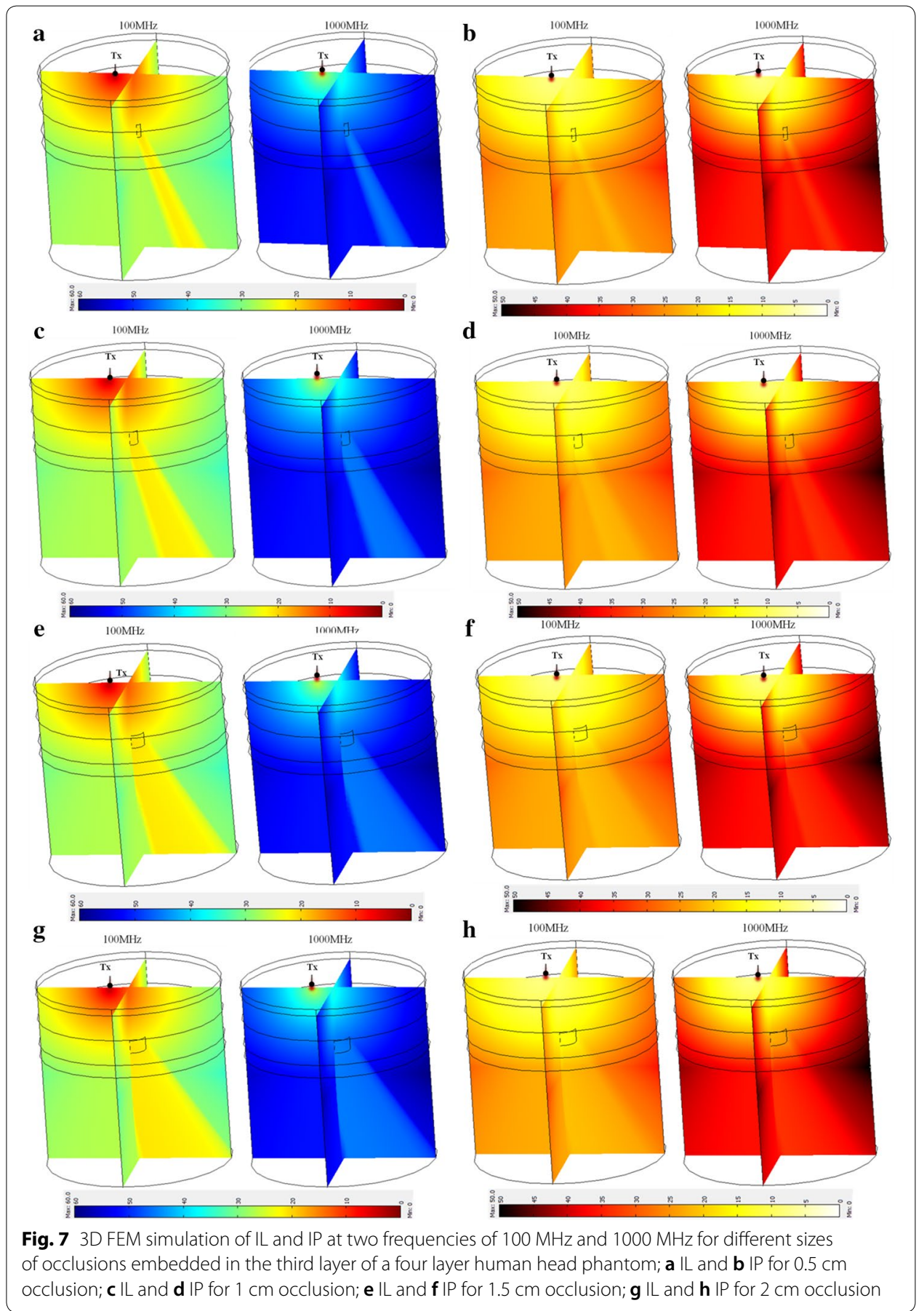

and largest occlusions, and that is shown when at $1000 \mathrm{MHz}$ of frequency modulation the difference between control and the smallest occlusion is $6-7 \mathrm{~dB}$ of IL and $4-5^{\circ}$ of IP for the $670 \mathrm{~nm}$ wavelength. This shows that for a small occlusion, the broadband frequency modulation system has an advantage of high sensitivity for detecting high oxygenated and de-oxygenated activities. In order to compare the experimental result to the numerical result a method using standard error calculation is used. The standard error, shown in Table 3, is based on integrated differences between the 

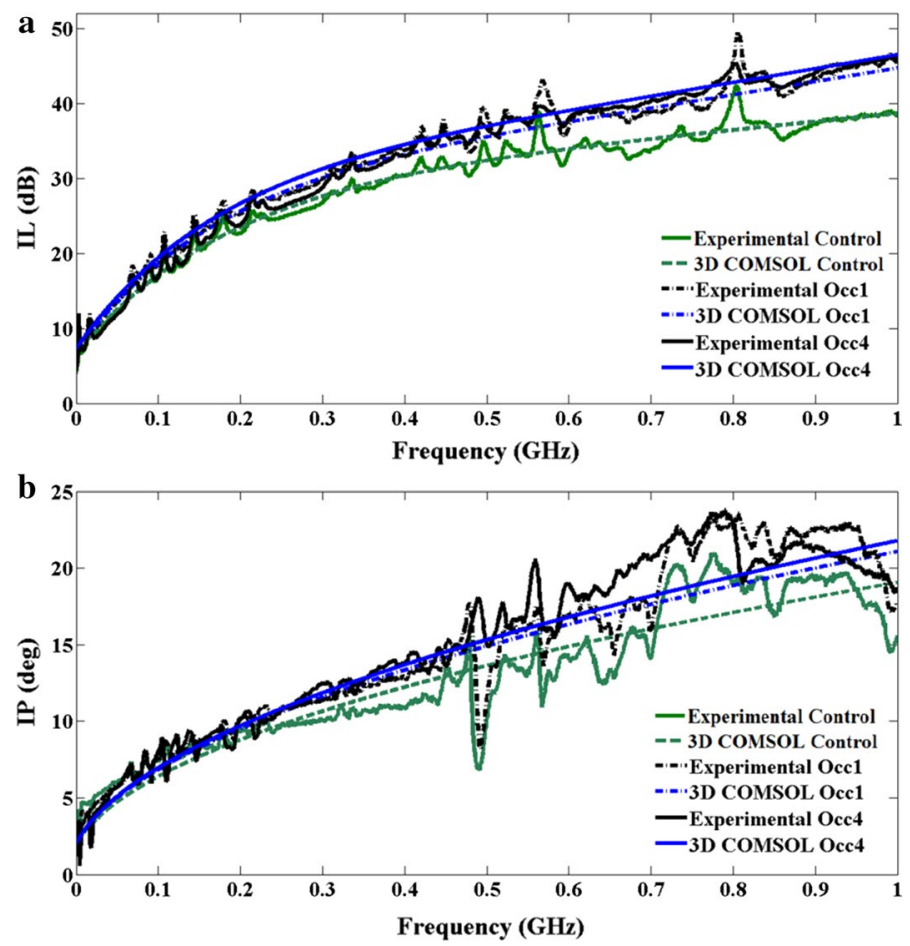

Fig. 8 Comparison of measured and FEM simulation results at NIR of $670 \mathrm{~nm}: \mathbf{a}$ IL, b IP from 30 to $1000 \mathrm{MHz}$

Table 3 Error between curved fitted experimental data and COMSOL 3D over various bandwidths

\begin{tabular}{|c|c|c|c|c|c|c|c|c|}
\hline \multirow[t]{2}{*}{ Wavelength } & \multicolumn{2}{|c|}{$\begin{array}{l}\text { Third layer w/ } \\
\text { occlusion } 1 \\
\text { Error \% }\end{array}$} & \multicolumn{2}{|c|}{$\begin{array}{l}\text { Third layer w/ } \\
\text { occlusion } 2 \\
\text { Error \% }\end{array}$} & \multicolumn{2}{|c|}{$\begin{array}{l}\text { Third layer w/ } \\
\text { occlusion } 3 \\
\text { Error \% }\end{array}$} & \multicolumn{2}{|c|}{$\begin{array}{l}\text { Third layer w/ } \\
\text { occlusion } 4 \\
\text { Error \% }\end{array}$} \\
\hline & IL & IP & IL & IP & IL & IP & IL & IP \\
\hline \multicolumn{9}{|l|}{670 nm } \\
\hline $30-1000 \mathrm{MHz}$ & 2.26 & 3.35 & 2.16 & 3.27 & 2.47 & 3.35 & 2.35 & 3.35 \\
\hline 30-300 MHz & 3.45 & 4.09 & 3.37 & 4.19 & 3.22 & 4.09 & 3.26 & 4.13 \\
\hline $300-500 \mathrm{MHz}$ & 5.85 & 5.35 & 5.44 & 5.42 & 5.64 & 5.35 & 5.63 & 5.68 \\
\hline $500-1000 \mathrm{MHz}$ & 4.74 & 4.65 & 4.24 & 4.49 & 4.09 & 4.74 & 4.51 & 4.07 \\
\hline
\end{tabular}

simulated FEM results for the multilayer phantom with occlusion and the curve fitted experimental results to the mathematical formulation of $a \sqrt{\text { frequency }}+b$ for each frequency data point divided by number of frequency data points. Mathematically the standard error is expressed as:

$$
\text { standard error }=\sqrt{\frac{\sum_{i=1}^{n}(\text { Curve fitted experimental data }-3 D \text { FEM simulation })^{2}}{n}}
$$

The low standard error values builds confidence in the 3D FEM modeling of inhomogeneous phantoms and facilitates the avenue to solve the inverse problem for accurate extraction of optical parameters that is required for tomographic imaging. 


\section{Early detection of TBI}

Results from previous sections support the task of accurate modeling of TBI using 3D FEM, and as an indication of this process it is observed that a change of slope of the resulted IL occurs when compared to the control IL. This indication is related to the understanding of frequency modulation concept of photons and flight of travel through the inhomogeneous media. Looking at Fig. 8 we see that at frequency around $300 \mathrm{MHz}$ we have a significant change in IL as compared to the surrounding frequencies. We are attributing this change in the sloe of IL to strong absorption of the photos by the occlusion. We are taking the advantage of this fact for identifying a hematoma occlusion. It should be noted that this specific frequency depends on the size and the location of the hematoma occlusion. Knowing the exact modulated frequency at which the most favorable path of photon is interacting with the embedded occlusion is of great interest, and it can be done using a threshold method of identifying a frequency-stamp at which the slope of IL is changed. It is important to understand that change of slope is observed in IL but it's not clearly observed in IP and that is adjustable due to the close values of $\mu_{s}^{\prime}$ for stacked layers of scalp, skull, and cortex. The slope of IP is increasingly high without change in slope and that is due to the high values of $\mu_{s}^{\prime}$. The threshold process is explained in Fig. 9, where first we compute $\Delta \mathrm{IL}$ between insertion loss due an embedded occlusion and when there is no occlusion. Then taking the first and second derivative of the resulted $\Delta \mathrm{IL}$ and locating the local minimum and zero frequency-axis cross over as the point of interaction between modulated photon and occlusion. Figure 10 shows the first and second derivative of $\Delta \mathrm{IL}$ and $\Delta \mathrm{IP}$ for wavelength of 670 and highlighted region shows the frequency-stamp at which the interaction is taking place. These frequency stamps are related to frequency modulated photon interaction with different sizes of occlusion as shown in Table 4.

\section{Discussions and conclusions}

This paper presents two important aspects of broadband frequency modulated fNIR system in terms of inverse problem of optical parameter extraction of multi-layer and inhomogeneous phantoms. We have demonstrated for the first time numerical modeling in 3D using FEM and its accuracy compared to the actual experimental results of phantom based human brain activity for with (abnormal) and without (normal)

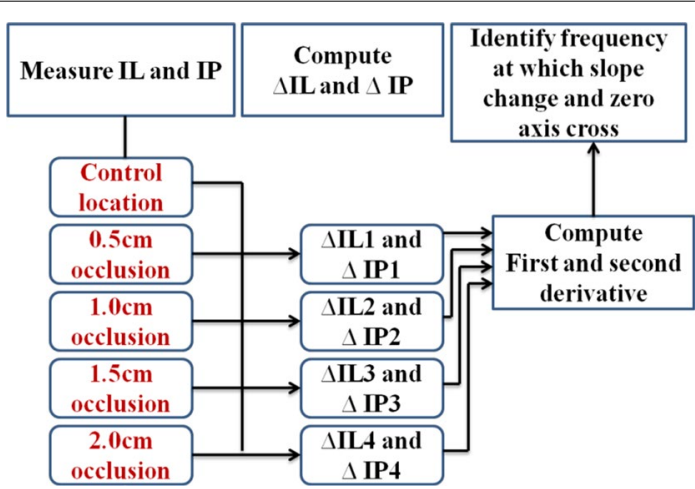

Fig. 9 Method of threshold detection based on first and second derivatives of $\Delta I L$ and $\Delta I P$ 


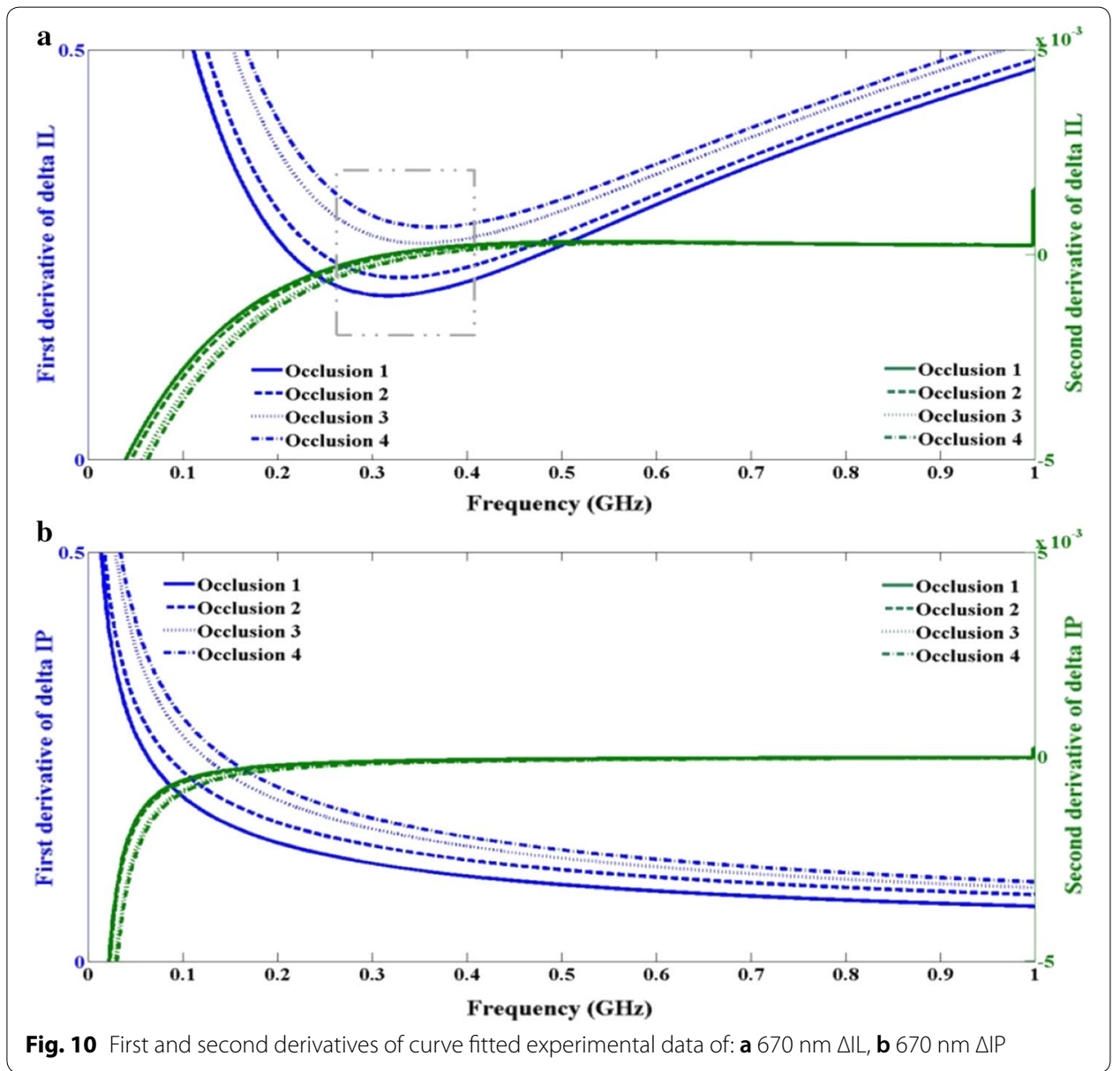

Table 4 Curve fitted experimental data threshold and frequency-stamp identification for different sizes of TBI

\begin{tabular}{lllll}
\hline Wavelength & $\begin{array}{l}\text { Occlusion } \\
\text { size }(\mathbf{c m})\end{array}$ & $\begin{array}{l}\text { Experimental } \\
\text { Freq. of } \mathbf{M F P}(\mathbf{M H z})\end{array}$ & $\begin{array}{l}\text { FEM modeling } \\
\text { Freq. of MFP }(\mathbf{M H z})\end{array}$ & $\begin{array}{l}\text { Error \% } \\
\text { Difference } \\
\text { between experimental } \\
\text { and modeling }\end{array}$ \\
\hline $670 \mathrm{~nm}$ & 0.5 & 376 & 371 & 1.3 \\
& 1.0 & 361 & 355 & 1.8 \\
& 1.5 & 343 & 337 & 1.8 \\
& 2.0 & 330 & 322 & 2.5 \\
\hline
\end{tabular}

hematoma, and its ability to successfully identify early signs of phantom based hematoma formation using highly sensitive signal processing methods. The accuracy of 3D numerical modeling is computed through the standard error calculation between numerical and curve fitted experimental result for wavelength of $670 \mathrm{~nm}$. A high confidence is developed with an error of less than $4 \%$, as rendered in Table 3, between simulation and measurement results over broadband measurements of 30-1000 MHz. The sensitivity of detecting different sizes of hematoma is clear with measurements for curve fitted experimental data and FEM predictions at different frequency-stamps 
at $670 \mathrm{~nm}$ wavelength, as shown in Table 4. FEM modeling shows a great deal of accuracy with modulated photons interacting with the smallest occlusion at $321 \mathrm{MHz}$ and a slope increase of $15-18 \mathrm{MHz}$ for each $0.5 \mathrm{~cm}$ increment in occlusion diameter. While curve fitted experimental data shows modulated photons interacting with the smallest occlusion optimally at $330 \mathrm{MHz}$ and frequency increments of 13-19 $\mathrm{MHz}$ for each $0.5 \mathrm{~cm}$ increment in diameter. These results are illustrated in Fig. 11, which based on the signal processing method described in previous sections, shows the frequency-stamp of each modulated photon interaction with occlusions for both curve fitted experimental data and FEM simulation results.

Extracted information of frequency stamp of the most favorable path of photons (MFP) gives insight to understanding the behavior of modulated photons in complex bio-media and supports the advantages of using broadband measurements. Frequency-stamp for both experimental and FEM simulation results are rendered in Table 4, and can be used to construct a conceptual modulated photon traveling in multi-layer phantom media as shown in Fig. 12. In fact, for different sizes of
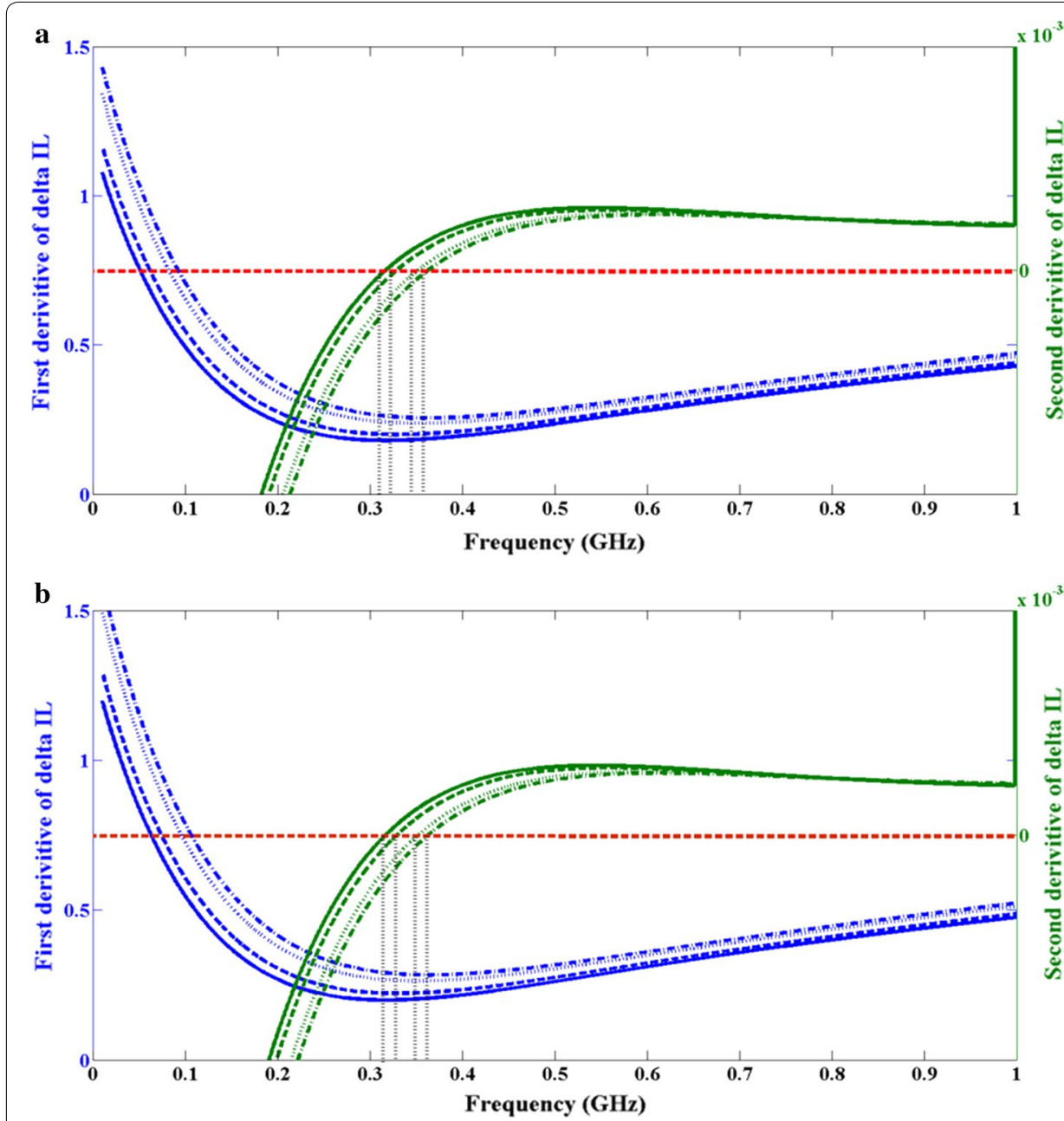

Fig. 11 First and second derivatives of $\Delta \mathrm{LL}$ between control and inhomogeneous third layer with different sizes of occlusions for $\mathbf{a}$ FEM simulation, and $\mathbf{b}$ curve fitted experimental data 


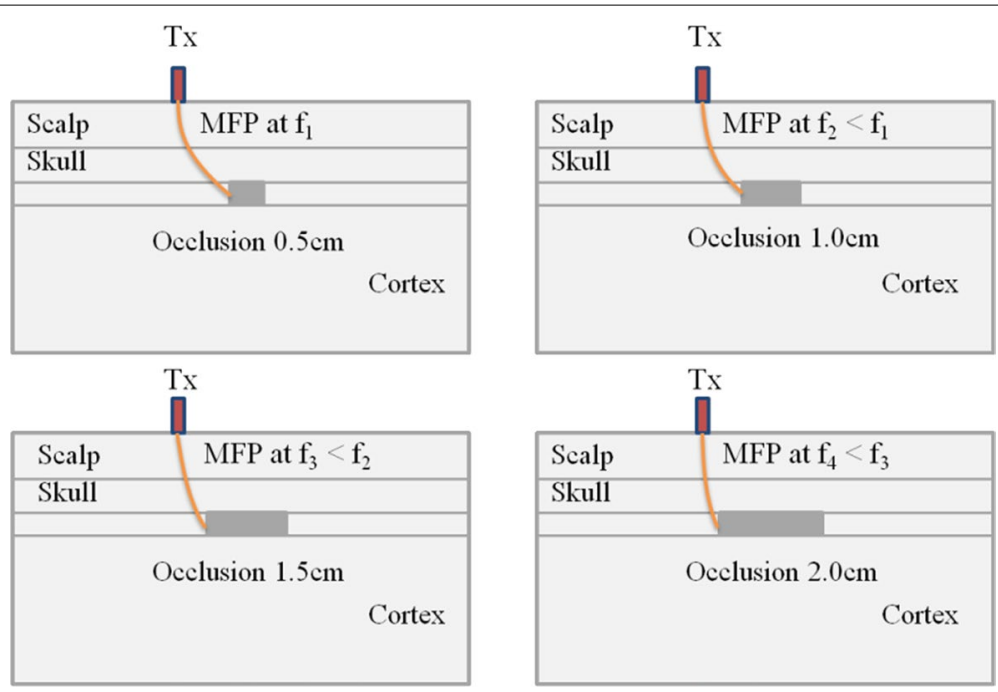

Fig. 12 Conceptual results of embedded occlusions and most favorable path for different modulating frequency of diffused photons

occlusions modulated wave of photons movement could be identified for a particular modulating frequency of diffused photons.

In conclusion, frequency modulated fNIR system shows a great potential in early detection of hematomas in multilayer brain phantom and have been validated as a high accurate tool through the use of numerical modeling. This was accomplished using 3D FEM and experimental measurements of IL and IP using broadband photon modulation on cases of normal and abnormal human head phantoms. Numerical modeling of 3D FEM shows a great deal of accuracy associated with homogenous [13] and inhomogeneous biological media [19], and can be used as a tool to perform inverse problem solving because of its low time consumption. Note this concept of 3D numerical modeling could be applied for any complex inhomogeneity of head structure and brain matter. First and second derivative for $\Delta \mathrm{IL}$ is reported here as a novel method of bio-marker to identify small size of hematoma with high accuracy.

Since this study is performed though one separation of $2 \mathrm{~cm}$ between an optical transmitter and receiver, it is important to indicate that the frequency associated with penetration depth and hematoma phantom location is not uniform to different optical transmitter and receiver separation and further adjustment is needed for different measurement topologies. Detection of small sized occlusions resembling formation of a hematoma and structure of each layer of the scalp and skull could be accomplished by taking advantage of the most favorable path of photon dependence on the modulating frequency of photons. This behavior is understood through the use of 3D FEM modeling and accuracy with the experimental measurements, as demonstrated for the first time in this paper. Frequency broadband measurements of IL and IP based on photon migration through multilayer human head phantom shows a promising breakthrough in detecting any high optical activity within certain depth beneath scalp.

\section{Authors' contributions}

ES: Made substantial/major contributions to conception and design, or acquisition of data, or analysis and interpretation of data. Given final approval of the version to be published. AD: Made contributions to conception and design, or 
acquisition of data, or analysis and interpretation of data. Given final approval of the version to be published. KP, AG: Involved in drafting/revising the manuscript and given final approval of the version to be published. All authors read and approved the final manuscript.

\section{Author details}

${ }^{1}$ Dept. of Electronics Engineering, College of Technological Studies, PAAET, Kuwait, Kuwait. ${ }^{2}$ Dept. of ECE, Drexel University, Philadelphia, PA 19104, USA. ${ }^{3}$ Eunice Kennedy Shriver National Institute of Child Health and Human Development, National Institutes of Health, Bethesda, MD 20892, USA. ${ }^{4}$ School of Biomedical Engineering, Science, and Health Systems, Drexel University, Philadelphia, PA 19104, USA.

\section{Acknowledgements}

Authors would like to acknowledge the support from PAAET and Drexel University RF Lab. This research is partly supported by the Center for Neuroscience and Regenerative Medicine (CNRM), and the Intramural Research Program (IRP) of Eunice Shriver National Institute of Child Health and Human Development (NICHD) of the National Institute of Health.

\section{Competing interests}

The authors declare that they have no competing interests.

\section{Availability of data and materials}

The datasets used and/or analyzed during the current study are available from the corresponding author on reasonable request.

\section{Consent for publication}

Not applicable.

Ethics approval and consent to participate

The manuscript does not have human participants, human data or human tissue. Therefore consent is not needed.

\section{Funding}

Partial funding from the Public Authority for Applied Education and Training (PAAET) was provided.

\section{Publisher's Note}

Springer Nature remains neutral with regard to jurisdictional claims in published maps and institutional affiliations.

Received: 14 July 2018 Accepted: 19 November 2018

Published online: 27 November 2018

\section{References}

1. Edlow BL, Wu O. Advanced neuroimaging in traumatic brain injury. Semin Neurol. 2012;32(04):374-400

2. Gaetz M. The neurophysiology of brain injury. Clin Neurophysiol. 2004;115(1):4-18.

3. Hukkelhoven CW, Steyerberg EW, Habbema JD, Farace E, Marmarou A, Murray GD, et al. Predicting outcome after traumatic brain injury: development and validation of a prognostic score based on admission characteristics. J Neurotrauma. 2005;22:1025-39.

4. Borg J, Holm L, Cassidy JD, Peloso PM, Carroll LJ, von Holst H, et al. Diagnostic procedures in mild traumatic brain injury: results of the WHO Collaborating Centre Task Force on Mild Traumatic Brain Injury. J Rehabil Med. 2004;36(suppl 43):61-75.

5. Mansouri C, Kashou NH. New window on optical brain imaging; medical development, simulations and applications. Selected topics on optical fiber technology; 2012.

6. Villringer A, Chance B. Non-invasive optical spectroscopy and imaging of human brain function. Trends Neurosci. 1997:20:435-42.

7. Yang Y, Liu H, Li X, Chance B. Low-cost frequency-domain photon migration instrument for tissue spectroscopy, oximetry, and imaging. Opt Eng. 1997;36(5):1562-9.

8. Durduran T, Choe R, Baker WB, Yodh AG. Diffuse optics for tissue monitoring and tomography. Rep Prog Phys. 2010;73(7):076801.

9. Riley JD, Amyot F, Pohida T, Pursley R, Ardeshirpour Y, Kainerstorfer JM, Najafizadeh L, Chernomordik V, Smith P, Smirniotopoulos J, Wassermann EM, Gandjbakhche AH. A hematoma detector-a practical application of instrumental motion as signal in near infra-red imaging. Biomed Opt Express. 2012;3(1):192-205.

10. Manseta K, et al. Development Challenges of brain functional monitoring using untethered broadband frequency modulated fNIR system. In: IEEE microwave photonics proceeding, MWP 2010, Montreal, Canada. pp. 295-9.

11. Manseta K, Khwaja AM, Sultan E, Daruwalla P, Pourrezaei K, Najafizadeh L, Gandjbakhche A, Daryoush AS. Untethered helmet mounted functional near infrared (fNIR) biomedical imaging?. In: Microwave symposium digest (MTT), 2011 IEEE MTT-S international, pp. 1, 4, 5-10 June 2011.

12. Sultan E, Manseta K, Khwaja A, Najafizadeh L, Gandjbakhche A, Pourrezaei K, Daryoush AS. Modeling and tissue parameter extraction challenges for free space broadband fNIR brain imaging systems. Proc SPIE. 2011;7902:790223.

13. Sultan E, Najafizadeh L, Gandjbakhche AH, Pourrezaei K, Daryoush A. Accurate optical parameter extraction procedure for broadband near-infrared spectroscopy of brain matter. J Biomed Opt. 2013;18(1):017008.

14. Hoshi Y, Shimada M, Sato C, Iguchi Y. Reevaluation of near-infrared light propagation in the adult human head: implications for functional near-infrared spectroscopy. J Biomed Opt. 2005;10(6):064032-064032-10. 
15. Tuchin V. Tissue optics: light scattering methods and instruments for medical diagnosis. 2nd ed. Bellingham: SPIE Press; 2007.

16. Zhou J, Bai J. The most favorable path method for the propagation of light in scattering media: a finite element solution. In: Engineering in medicine and biology society, 2001. Proceedings of the 23rd annual international conference of the IEEE, vol. 4, pp. 3203, 3206. 2001.

17. Arridge SR, Schweiger M, Hiraoka M, Delpy DT. A finite element approach for modeling photon transport in tissue. Med Phys. 1993;20:299.

18. Troy TL, Thenadil SN. Optical properties of human skin in the near infrared wavelength range of 1000 to $2200 \mathrm{~nm}$. J Biomed Opt. 2001;6:167-76.

19. Sultan E, Pourrezaei K, Gandjbakhche AH, Daryoush A. 3D numerical modeling and its experimental verifications for an inhomogeneous head phantom using broadband fNIR system. Int J Numer Methods Biomed Eng. 2014;30:353-64.

- fast, convenient online submission

- thorough peer review by experienced researchers in your field

- rapid publication on acceptance

- support for research data, including large and complex data types

- gold Open Access which fosters wider collaboration and increased citations

- maximum visibility for your research: over 100M website views per year

At BMC, research is always in progress.

Learn more biomedcentral.com/submissions 